\title{
Condorcet versus participation criterion in social welfare rules
}

Citation for published version (APA):

Can, B., Ergin, E., \& Pourpouneh, M. (2017). Condorcet versus participation criterion in social welfare rules. Maastricht University, Graduate School of Business and Economics. GSBE Research Memoranda No. 020 https://doi.org/10.26481/umagsb.2017020

Document status and date:

Published: 14/09/2017

DOI:

10.26481/umagsb.2017020

Document Version:

Publisher's PDF, also known as Version of record

\section{Please check the document version of this publication:}

- A submitted manuscript is the version of the article upon submission and before peer-review. There can be important differences between the submitted version and the official published version of record.

People interested in the research are advised to contact the author for the final version of the publication, or visit the DOI to the publisher's website.

- The final author version and the galley proof are versions of the publication after peer review.

- The final published version features the final layout of the paper including the volume, issue and page numbers.

Link to publication

\footnotetext{
General rights rights.

- You may freely distribute the URL identifying the publication in the public portal. please follow below link for the End User Agreement:

www.umlib.nl/taverne-license

Take down policy

If you believe that this document breaches copyright please contact us at:

repository@maastrichtuniversity.nl

providing details and we will investigate your claim.
}

Copyright and moral rights for the publications made accessible in the public portal are retained by the authors and/or other copyright owners and it is a condition of accessing publications that users recognise and abide by the legal requirements associated with these

- Users may download and print one copy of any publication from the public portal for the purpose of private study or research.

- You may not further distribute the material or use it for any profit-making activity or commercial gain

If the publication is distributed under the terms of Article $25 \mathrm{fa}$ of the Dutch Copyright Act, indicated by the "Taverne" license above, 


\section{Maastricht University}

Burak Can, Emre Ergin, Mohsen Pourpouneh

Condorcet versus participation criterion in social welfare rules

$\mathrm{RM} / 17 / 020$

\section{GSBE}

Maastricht University School of Business and Economics

Graduate School of Business and Economics

P.O Box 616

NL-6200 MD Maastricht

The Netherlands 


\title{
Condorcet versus participation criterion in social welfare rules
}

\author{
Burak Can* Emre Ergin $^{\dagger} \quad$ Mohsen Pourpouneh $^{\ddagger}$
}

August 28, 2017

\begin{abstract}
Moulin (1988) shows that there exists no social choice rule, that satisfies the following two criteria at the same time: the Condorcet criterion and the participation criterion, a.k.a., No Show Paradox. We extend these criteria to social welfare rules, i.e., rules that choose rankings for each preference profile. We show that the impossibility does not hold, and one particular rule, the Kemeny rule satisfies both the Condorcet and the participation criteria.
\end{abstract}

Keywords: Condorcet criterion, participation criterion, social choice rules, social welfare rules.

${ }^{*}$ Corresponding author: Department of Economics, School of Business and Economics, Maastricht University, P.O. Box 616, 6200 MD Maastricht, the Netherlands, e-mail: b.can@maastrichtuniversity.nl, Phone number: +31 43 3883807. This work is partly financed by the Netherlands Organisation for Scientific Research (NWO) under the grant with project nr. 451-13-017 (VENI, 2014): "Policy Design in Dynamic Matching Markets". The support of the NWO, therefore, is gratefully acknowledged.

${ }^{\dagger}$ Maastricht University, Department of Economics. E-mail: ergin@maastrichtuniversity.nl.

${ }^{\ddagger}$ Department of Mathematical Science, Sharif University of Technology and Department of Quantitative Economics, Maastricht University, e-mail: m_pourpouneh@mehr.sharif.ir 


\section{Introduction}

Two of the most well-known criteria for social choice rules are the Condorcet criterion and the participation criterion. The former, introduced by Marquis de Condorcet (1765), requires that if there is a candidate who beats all others in a pairwise comparison then it should be the unique winner. The rules that satisfy this property are also called Condorcet methods. The latter, introduced in Fishburn and Brams (1983) as No Show Paradox, requires that attending to an election should be somewhat incentive compatible. To be more precise, for any voter, participating an election should not change the result into a less desirable outcome ${ }^{1}$ for that voter. Moulin (1988) shows that the Condorcet criterion and the participation criterion are incompatible ${ }^{2}$. To put it differently, there exists no Condorcet method which satisfies the participation criterion, hence they are all vulnerable to No Show Paradox.

We extend these two criteria to social welfare rules, i.e., rules that assign rankings of alternatives to each preference profile instead of a winning alternative. Thereafter we investigate whether the incompatibility between the Condorcet and the participation concepts still exists for the social welfare rules. Young and Levenglick (1978) introduce a natural extension of the Condorcet concept to social welfare rules. To the best of our knowledge, an extension of the participation criterion to social welfare rules does not exist.

In this paper, we propose a weaker version of the Condorcet concept developed in Young and Levenglick (1978) as the Condorcet criterion. We also introduce a natural extension of the participation criterion for social welfare rules. As expected many social welfare rules fail to satisfy both at the same time. However, we find that one particular rule satisfies both criteria, the Kemeny ${ }^{3}$ rule. Hence we show that the incompatibility, introduced in Moulin (1988), between the two concepts does not exist on the domain of social welfare rules.

The rest of this paper is organized as follows. In Section 2, we define the notation and the model. In Section 3, we introduce the Condorcet and the participation criteria for social welfare rules. Section 4 discusses various social welfare rules and shows that the Kemeny rule satisfies both criteria. Section 5, concludes the paper with discussion and open questions.

\footnotetext{
${ }^{1}$ For further results related to participation criteria or No Show Paradox, see Pérez (2001); Felsenthal and Nurmi (2016); Brandt et al. (2016); Núñez and Sanver (2017).

${ }^{2}$ The incompatibility holds so long as there are at least four alternatives and twenty-five individuals.

${ }^{3}$ Kemeny rule, also known as Kemeny-Young method, is introduced in Young and Levenglick (1978). See also Can and Storcken (2013b) for another characterization.
} 


\section{Model}

Let $N=\{1,2, \ldots, n\}$ be a non-empty and finite set of $n$ individuals and $A$ be a non-empty and finite set of $m$ alternatives. Let $\mathbb{L}$ denote the set of all possible linear orders (strict rankings) over $A$, i.e., complete, transitive, and antisymmetric binary relations over $A$. For a generic $R \in \mathbb{L}$, and any two alternatives $a, b \in A,(a, b) \in R$ is interpreted as " $a$ is at least as good as $b$ at $R$ ". In case $a$ and $b$ are two distinct alternatives the relation is strict. To simplify notation, we write $a b \in R$ instead of $(a, b) \in R$. Similarly $R=a b c d$ denotes a full ranking where $R=\{a a, a b, a c, a d, b b, b c, b d, c c, c d, d d\}$.

A (preference) profile $p=(p(1), \ldots, p(n))$ is an $n$-tuple vector in $\mathbb{L}^{N}$, where $p(i)$ is the preference ordering of individual $i$ over $A$. Given $p \in \mathbb{L}^{N}$, the profile of all individuals except $i$ is denoted by $p_{-i} \in \mathbb{L}^{N \backslash\{i\}}$, i.e., $p_{-i}=(p(1), \ldots, p(i-1), p(i+1), \ldots, p(n))$. We denote the number of individuals who prefer $a$ to $b$ minus the number of individuals who prefer $b$ to $a$, by $n_{a b}(p)$. Formally, $n_{a b}(p)=|\{i \in N: a b \in p(i)\}|-|\{i \in N: b a \in p(i)\}|$. We define the corresponding tournament $T_{p}$ of a profile $p$ by $T_{p}=\left\{a b \in A \times A: n_{a b}(p)>0\right\}$. Moreover, we say $a$ beats $b$ whenever $a b \in T_{p}$.

We denote the utility of an agent $i$ over the set of alternatives by $u_{i}: A \rightarrow \mathbb{R}$ such that $u_{i}(a) \geq u_{i}(b)$ if and only if $\rho(a, p(i)) \leq \rho(b, p(i))$ where for any $a \in A, \rho(a, p(i))=\mid x \in A$ : $x a \in p(i) \mid$, i.e., the rank of $a$ at $p(i)$. Similarly, we denote the utility of an agent $i$ over the set of rankings by $U_{i}: \mathbb{L} \rightarrow \mathbb{R}$ such that $U_{i}(R) \geq U_{i}\left(R^{\prime}\right)$ if and only if $\delta(p(i), R) \leq \delta\left(p(i), R^{\prime}\right)$ where for any $R, R^{\prime} \in \mathbb{L}, \delta\left(R, R^{\prime}\right)=\left|R \backslash R^{\prime}\right|+\left|R^{\prime} \backslash R\right|$, i.e., the Kemeny distance ${ }^{4}$ between $R$ and $R^{\prime}$. Note that, as $R$ and $R^{\prime}$ are both linear orders, we have, $\left|R \backslash R^{\prime}\right|=\left|R^{\prime} \backslash R\right|$. Furthermore $\left|R \backslash R^{\prime}\right|=|R|-\left|R \cap R^{\prime}\right|=m(m-1)-\left|R \cap R^{\prime}\right|$. Therefore, the Kemeny distance can also be denoted by $\delta\left(R, R^{\prime}\right)=2\left|R \backslash R^{\prime}\right|=2\left|R^{\prime} \backslash R\right|=2 m(m-1)-2\left|R \cap R^{\prime}\right|$.

\section{Condorcet criteria vs participation criteria}

We first remind the reader of the formal definitions of the Condorcet and the participation criteria on social choice rules. Thereafter we extend these concepts to social welfare rules.

\subsection{Social choice rules}

A social choice rule $\psi: \mathbb{L}^{N} \rightarrow 2^{A} \backslash \emptyset$, maps every profile $p$ to a non-empty subset of alternatives.

Let $p \in \mathbb{L}^{N}$ be a profile, then an alternative $a \in A$ is called the Condorcet winner whenever $n_{a b}(p)>0$, for all $b \in A \backslash\{a\}$.

\footnotetext{
${ }^{4}$ Kemeny distance is introduced and characterized in Kemeny (1959), an improved characterization can be found in Can and Storcken (2013a).
} 
Definition 1 (Condorcet Criterion (CCC)) A social choice rule $\psi$ satisfies Condorcet criterion for choice rules whenever for all $p \in \mathbb{L}^{N}$, with a Condorcet winner $a \in A$, we have $\psi(p)=a$.

Definition 2 (Participation Criterion (PCC)) A social choice rule $\psi$ satisfies participation criterion for choice rules whenever for all $p \in \mathbb{L}^{N}$, and for all $i \in N$,

$$
\max _{a \in \psi(p)} u_{i}(a) \geq \max _{b \in \psi\left(p_{-i}\right)} u_{i}(b)
$$

That is, the best outcome of the social choice rule for each individual when he participates is at least as good as the best outcome when he does not participate.

\subsection{Social welfare rules}

A social welfare rule $\varphi: \mathbb{L}^{N} \rightarrow 2^{\mathbb{L}} \backslash \emptyset$, maps every preference profile $p$ to a non-empty subset of linear orders. Typically the Arrovian framework considers the range of welfare rules to be weak orders, i.e., the outcome is a single ranking that might admit ties. Consider for instance the cyclical profile with three individuals and three alternatives, $p=(a b c, b c a, c a b)$. The Borda score of each alternative equals 3. Hence the outcome of Borda welfare function is a single weak order, $\varphi^{\text {Borda }}(p)=a \sim b \sim c$, where " $\sim$ " denotes the indifference. However, there are rules that aggregate preference profiles into multiple strict rankings without ties such as the Kemeny rule. The Kemeny rule would assign the following three rankings as the outcome to the aforementioned profile, $\varphi^{\text {Kemeny }}(p)=\{a b c, b c a, c a b\}$.

Note that, any weak order can be extended to a set of strict rankings, by simple breaking all ties neutrally. Therefore each Arrovian social welfare function can also be treated as a social welfare correspondence assigning possibly multiple ${ }^{5}$ strict rankings as the outcome. This does not only give comparability across rules, but also provides a unified axiomatic framework to analyse all ranking aggregators. In what follows we shall consider social welfare rules that assign a set of strict rankings to each preference profile. In this context the outcome of the Borda rule in the example above will be all possible linear extensions of the weak order $\varphi^{B o r d a}(p)=a \sim b \sim c$, i.e., $\varphi^{B o r d a}(p)=\{a b c, a c b, b a c, b c a, c a b, c b a\}$.

Let $p \in \mathbb{L}^{N}$ be a profile, then a ranking $R \in \mathbb{L}$ is called the Condorcet ranking whenever $n_{a b}(p)>0$ for all distinct $a b \in R$.

Definition 3 (Condorcet Criterion $(\boldsymbol{C C W})$ ) A social welfare rule $\varphi$ satisfies Condorcet criterion whenever for all $p \in \mathbb{L}^{N}$, with a Condorcet ranking $R \in \mathbb{L}$, we have $\varphi(p)=R$.

\footnotetext{
${ }^{5}$ For multiplicity in social choice rules and related participation criteria, see Jimeno et al. (2009).
} 
Definition 4 (Participation Criterion $(\boldsymbol{P C W})$ ) A social welfare rule $\varphi$ satisfies participation criterion whenever for all $p \in \mathbb{L}^{N}$, and for all $i \in N$,

$$
\max _{R \in \varphi(p)} U_{i}(R) \geq \max _{R^{\prime} \in \varphi\left(p_{-i}\right)} U_{i}\left(R^{\prime}\right) .
$$

That is, the best outcome of the social welfare rule for each individual when he participates is at least as good as the best outcome when he does not participate.

\section{Results}

In this section, we investigate the Copeland, Minimax, Borda and Kemeny welfare rules to see whether they satisfy the Condorcet criterion $(C C W)$ and participation criterion $(P C W)$.

\subsection{Copeland rule}

According to the Copeland ${ }^{6}$ social choice rule, the winner is the candidate(s), which has the highest Copeland score. The Copeland score of a candidate is the number of alternatives that are beaten by that candidate.

A natural extension of this social choice rule to the social welfare domain is by ordering the alternatives based on their Copeland scores. Formally, let $\operatorname{Cscore}(a, p)=\mid\{b \in A$ : $\left.a b \in T_{p}\right\} \mid$. The Copeland rule is defined as follows,

$$
\varphi^{\text {Copeland }}(p)=\{R \in \mathbb{L}: C(p) \subseteq R\}
$$

where $C(p)=\{a b \in A \times A: \operatorname{Cscore}(a, p) \geq C$ score $(b, p)\}$ is the ordering of the alternatives with respect to their scores. The Copeland rule chooses all linear extensions of this (possibly) weak ordering.

Proposition 1 Copeland rule does not satisfy PCW.

Proof. See Appendix A.1.

Proposition 2 Copeland rule satisfies $C C W$.

Proof. See Appendix A.2.

\footnotetext{
${ }^{6}$ Copeland (1951)
} 


\subsection{Minimax rule}

According to the Minimax ${ }^{7}$ social choice rule, the winner is the candidate(s), which has the lowest Minimax score. The Minimax score of a candidate is the maximum number of individuals who prefer another particular alternative to that candidate.

A natural extension of this social choice rule to the social welfare domain is by ordering the alternatives based on their Minimax scores. Formally, let $M \operatorname{score}(a, p)=\max _{b \in A \backslash\{a\}} \mid\{i \in$ $N: b a \in p(i)\} \mid$. The Minimax rule is defined as follows,

$$
\varphi^{\operatorname{Minimax}}(p)=\{R \in \mathbb{L}: M(p) \subseteq R\},
$$

where $M(p)=\{a b \in A \times A: \operatorname{Mscore}(a, p) \leq \operatorname{Mscore}(b, p)\}$ is the ordering of alternatives with respect to their scores. The Minimax rule chooses all linear extensions of this (possibly) weak ordering.

Proposition 3 Minimax does not satisfy PCW.

Proof. See Appendix B.1.

Proposition 4 Minimax does not satisfy $C C W$.

Proof. See Appendix B.2.

\subsection{Borda rule}

According to the Borda ${ }^{8}$ social choice rule, the winner is the candidate(s), which has the highest Borda score. Roughly speaking, Borda score of a candidate is the total number of alternatives that are less desirable than that candidate, across all individuals.

A natural extension of this social choice rule to the social welfare domain is by ordering the alternatives based on their Borda scores. Formally, let $\operatorname{Bscore}(a, p)=\sum_{i \in N} \mid\{b \in$ $A \backslash\{a\}: a b \in p(i)\} \mid$. The Borda rule is defined as follows,

$$
\varphi^{B o r d a}(p)=\{R \in \mathbb{L}: B(p) \subseteq R\},
$$

where $B(p)=\{a b \in A \times A: \operatorname{Bscore}(a, p) \geq B$ score $(b, p)\}$ is the ordering of alternatives with respect to their scores. The Borda rule chooses all linear extensions of this (possibly) weak ordering.

Proposition 5 Borda satisfies $P C W$.

\footnotetext{
${ }^{7}$ Black et al. (1958); Simpson (1969); Kramer (1977)

${ }^{8}$ de Borda (1953); Black et al. (1958)
} 
Proof. See Appendix C.1.

Proposition 6 Borda does not satisfy $C C W$.

Proof. See Appendix C.2.

\subsection{Kemeny rule}

The notion of Kemeny distance between two linear orders can be extended to the distance between a linear order and a profile. That is the distance of a linear order to a profile is the sum of the Kemeny distances of the linear order to each individual's preference. The outcome of the Kemeny rule is the set of linear orders which minimizes this distance to the profile. Formally, the Kemeny rule is defined as follows,

$$
\varphi^{\text {Kemeny }}(p)=\left\{R \in \mathbb{L}: \delta(p, R) \leq \delta\left(p, R^{\prime}\right) \text { for all } R^{\prime} \in \mathbb{L}\right\},
$$

in which $\delta(p, R)=\sum_{i \in N} \delta(p(i), R)$.

Proposition 7 Kemeny Rule satisfies $P C W$.

Proof. Let $p$ be a profile. Let $\bar{R} \in \underset{R \in \varphi^{\text {Kemeny }}(p)}{\operatorname{argmax}} U_{i}(R)$ and $\hat{R} \in \underset{R \in \varphi^{\text {Kemeny }}\left(p_{-i}\right)}{\operatorname{argmax}} U_{i}(R)$ for any agent $i$ in the set of individuals. Since $\bar{R} \in \varphi^{\text {Kemeny }}(p)$, by definition of the Kemeny rule, $\sum_{i \in N} \delta(p(i), \bar{R}) \leq \sum_{i \in N} \delta(p(i), \hat{R})$, hence

$$
\sum_{j \in N \backslash\{i\}} \delta(p(j), \bar{R})+\delta(p(i), \bar{R}) \leq \sum_{j \in N \backslash\{i\}} \delta(p(j), \hat{R})+\delta(p(i), \hat{R})
$$

Since $\hat{R}=\varphi^{\text {Kemeny }}\left(p_{-i}\right)$, by definition of the Kemeny rule,

$$
\sum_{j \in N \backslash\{i\}} \delta\left(p_{-i}(j), \hat{R}\right) \leq \sum_{j \in N \backslash\{i\}} \delta\left(p_{-i}(j), \bar{R}\right) .
$$

As $p(j)=p_{-i}(j)$ for all $j \in N \backslash\{i\}$, then above equation is equivalent to,

$$
\sum_{j \in N \backslash\{i\}} \delta(p(j), \hat{R}) \leq \sum_{j \in N \backslash\{i\}} \delta(p(j), \bar{R}) .
$$

Adding $\delta(p(i), \hat{R})$ to both sides of Equation 2 results in,

$$
\sum_{j \in N \backslash\{i\}} \delta(p(j), \hat{R})+\delta(p(i), \hat{R}) \leq \sum_{j \in N \backslash\{i\}} \delta(p(j), \bar{R})+\delta(p(i), \hat{R}) .
$$


Combining Equations 1 and 3 results in,

$$
\sum_{j \in N \backslash\{i\}} \delta(p(j), \bar{R})+\delta(p(i), \bar{R}) \leq \sum_{j \in N \backslash\{i\}} \delta(p(j), \bar{R})+\delta(p(i), \hat{R}) .
$$

Therefore, $\delta(p(i), \bar{R}) \leq \delta(p(i), \hat{R})$ which completes the proof.

Proposition 8 Kemeny satisfies $C C W$.

Proof. Let $p$ be a profile such that $R$ is the Condorcet ranking. Lemma 12 in Can and Storcken (2013b) shows that the outcome of the Kemeny rule coincides with the linear extensions of the pairwise majority relation, i.e., $T_{p}$. As this equals the unique Condorcet ranking $R$, we conclude $R=\varphi^{\text {Kemeny }}(p)$.

\section{Conclusion}

This paper extends the two appealing, albeit conflicting, criteria on social choice rules to social welfare rules. We show that several welfare rules fail to satisfy both of these criteria at the same time. However, we also find that the Kemeny rule satisfies both of these extended criteria. This shows that there is at least one welfare rule that is incentive compatible, i.e., voters do not have a reason to abstain, and at the same time respecting majority opinion in a Condorcet consistent fashion.

We show that rules that have both social choice and social welfare versions might behave differently in these two domains. Copeland rule satisfies the Condorcet criteria both as a (social) choice and as a (social) welfare rule, but both versions fail respective participation criteria. The Minimax rule satisfies Condorcet criteria as a choice rule but fails it as a welfare rule. Both versions fail respective participation criteria too. Borda rule fails the Condorcet criteria both as a choice and as a welfare rule but both versions satisfy the respective participation criteria. Interestingly, the Kemeny rule satisfies Condorcet criteria both as a choice and as a welfare rule, yet only the welfare version satisfies the corresponding participation criterion. Table 1 shows a summary of the results.

We think the question is still very much open especially because it is not clear whether there are more social welfare rules that satisfy both of these criteria, i.e., $C C W$ and $P C W$. It might as well be that these, together with other axioms, characterize the Kemeny rule, or perhaps a class of rules, with similar characteristics, e.g., Slater rule ${ }^{9}$ rule.

\footnotetext{
${ }^{9}$ See Slater (1961); Nurmi (2014).
} 
Table 1: Summary of the results.

\begin{tabular}{ccccc}
\hline Rule & $C C C$ & $P C C$ & $C C W$ & $P C W$ \\
\hline \hline Copeland & $\checkmark$ & $\times$ & $\checkmark$ & $\times$ \\
Minimax & $\checkmark$ & $\times$ & $\times$ & $\times$ \\
Borda & $\times$ & $\checkmark$ & $\times$ & $\checkmark$ \\
Kemeny & $\checkmark$ & $\times$ & $\checkmark$ & $\checkmark$ \\
\hline$\checkmark$ : satisfy, $\times$ : not satisfy & &
\end{tabular}

\section{Acknowledgement}

Part of this research has been done when Mohsen Pourpouneh was a visiting Ph.D. student of Sharif University of Technology under the supervision of A. Daneshgar and R. Ramezanian at Maastricht University.

\section{References}

Black, D., R. A. Newing, I. McLean, A. McMillan, and B. L. Monroe (1958). The theory of committees and elections.

Brandt, F., C. Geist, and D. Peters (2016). Optimal bounds for the no-show paradox via sat solving. Mathematical Social Sciences.

Can, B. and A. Storcken (2013a). A re-characterization of the kemeny distance.

Can, B. and T. Storcken (2013b). Update monotone preference rules. Mathematical Social Sciences 65(2), 136-149.

Condorcet, M. (1765). Marquis de (1785). Essai sur lapplication de lanalyse à la probabilité des décisions rendues à la pluralité des voix.

Copeland, A. H. (1951). A reasonable social welfare function. In Mimeographed notes from a Seminar on Applications of Mathematics to the Social Sciences, University of Michigan.

de Borda, J. C. (1953). Memoire sur les elections au scrutin, 1781. Histoire de lAcademie Royale des Sciences, Paris.

Felsenthal, D. S. and H. Nurmi (2016). Two types of participation failure under nine voting methods in variable electorates. Public Choice 168(1-2), 115-135. 
Fishburn, P. C. and S. J. Brams (1983). Paradoxes of preferential voting. Mathematics Magazine 56(4), 207-214.

Jimeno, J. L., J. Pérez, and E. García (2009). An extension of the moulin no show paradox for voting correspondences. Social Choice and Welfare 33(3), 343-359.

Kemeny, J. G. (1959). Mathematics without numbers. Daedalus 88(4), 577-591.

Kramer, G. H. (1977). A dynamical model of political equilibrium. Journal of Economic Theory 16(2), 310-334.

Moulin, H. (1988). Condorcet's principle implies the no show paradox. Journal of Economic Theory 45(1), 53-64.

Núñez, M. and M. R. Sanver (2017). Revisiting the connection between the no-show paradox and monotonicity. Mathematical Social Sciences 86, 59-67.

Nurmi, H. (2014). Are we done with preference rankings? if we are, then what? Operations Research and Decisions $24(4), 63-74$.

Pérez, J. n. (2001). The strong no show paradoxes are a common flaw in condorcet voting correspondences. Social Choice and Welfare 18, 601-616.

Simpson, P. B. (1969). On defining areas of voter choice: Professor tullock on stable voting. The Quarterly Journal of Economics 83(3), 478-490.

Slater, P. (1961). Inconsistencies in a schedule of paired comparisons. Biometrika 48(3/4), 303-312.

Young, H. P. and A. Levenglick (1978). A consistent extension of condorcets election principle. SIAM Journal on applied Mathematics 35 (2), 285-300. 


\section{A Proofs for Copeland rule}

\section{A.1 Copeland does not satisfy $P C W$.}

Proof of Proposition 1. Consider the following profile $p$ with twenty-one individuals and the set of alternatives $A=\{a, b, c, d\}$.

\begin{tabular}{lllll}
3 & 3 & 4 & 5 & 6 \\
\hline \hline$a$ & $a$ & $b$ & $d$ & $b$ \\
$d$ & $d$ & $c$ & $b$ & $d$ \\
$b$ & $c$ & $a$ & $c$ & $a$ \\
$c$ & $b$ & $d$ & $a$ & $c$ \\
\hline
\end{tabular}

It can be verified that $T_{p}=\{d a, d b, d c, b a, b c, a c\}$ and hence $\varphi^{\text {Copeland }}(p)=\{d b a c\}$. Now assume the agent $i$ with the preference ranking $p(i)=b d a c$, decides not to participate. Then it can be verified that $T_{p_{-i}}=\{d b, d c, b a, b c, a c\}$ and therefore $\varphi^{\text {Copeland }}\left(p_{-i}\right)=\{b d a c, d b a c\}$. As $b d a c$ maximizes the utility of individual $i$ and $U_{i}(b d c a)>U_{i}(d b a c)$, it follows that Copeland does not satisfy $P C W$.

\section{A.2 Copeland satisfies $C C W$.}

Proof of Proposition 2. Let $p$ be a profile and let $R=a_{1} a_{2} \ldots a_{m}$ be the Condorcet ranking of the profile. By definition of Condorcet ranking $n_{a_{i} a_{k}}>0$ for all $i \in\{1, \ldots, m\}$ and $k \in\{i+1, \ldots, m\}$. Since Cscore $\left(a_{i}, p\right)=\left|a_{k} \in A: a_{i} a_{k} \in T_{p}\right|=\mid\left\{a_{i+1}, a_{i+2}, \ldots\right.$, $\left.a_{m}\right\} \mid=m-i$, then by definition of Copeland rule $a_{1} a_{2} \ldots a_{m}$ will be the outcome which coincides with the Condorcet ranking.

\section{B Proofs for Minimax rule}

\section{B.1 Minimax does not satisfy $P C W$.}

Proof of Proposition 3. Consider the following profile $p$ with seventeen individuals and the set of alternatives $A=\{a, b, c, d\}$.

\begin{tabular}{cccccccc}
3 & 2 & 2 & 2 & 2 & 2 & 2 & 2 \\
\hline \hline$a$ & $d$ & $a$ & $c$ & $b$ & $c$ & $d$ & $b$ \\
$b$ & $c$ & $d$ & $b$ & $d$ & $a$ & $c$ & $a$ \\
$c$ & $a$ & $b$ & $a$ & $a$ & $b$ & $a$ & $d$ \\
$d$ & $b$ & $c$ & $d$ & $c$ & $d$ & $b$ & $c$ \\
\hline
\end{tabular}


The above profile can be summarized in the following "profile matrix" $\bar{p}$ where the cell $x y$ in $\bar{p}$ denotes the number of individuals that prefer $x$ to $y$.

$$
\bar{p}=\begin{gathered}
a \\
b \\
c \\
d
\end{gathered}\left[\begin{array}{cccc}
a & b & c & d \\
0 & 11 & 9 & 11 \\
6 & 0 & 9 & 11 \\
8 & 8 & 0 & 7 \\
6 & 6 & 10 & 0
\end{array}\right]
$$

It can be verified that $\varphi^{\operatorname{Minimax}}(p)=\{a c b d, a c d b\}$. Now assume the agent $i$ with the preference ranking $p(i)=a b c d$ decides not to participate. Then it can be verified that the profile matrix would be as the following,

$$
\bar{p}_{-i}=\begin{gathered}
a \\
a \\
b \\
d
\end{gathered}\left[\begin{array}{cccc}
0 & 10 & 8 & 10 \\
6 & 0 & 8 & 10 \\
8 & 8 & 0 & 6 \\
6 & 6 & 10 & 0
\end{array}\right]
$$

It can be verified that $\varphi^{\operatorname{Minimax}}\left(p_{-i}\right)=\{a b c d, a b d c, a c b d, a c d b, a d b c, a d c b\}$. It is easy to see that $a c b d \in \underset{R \in \varphi^{\text {Minimax }}(p)}{\operatorname{argmax}} U_{i}(R)$ and $a b c d \in \underset{R \in \varphi^{\operatorname{Minimax}}\left(p_{-i}\right)}{\operatorname{argmax}} U_{i}(R)$. Since $U_{i}(a b c d)>$ $U_{i}(a c b d)$, it follows that Minimax does not satisfy $P C W$.

\section{B.2 Minimax does not satisfy $C C W$.}

Proof of Proposition 4. Let $A=\{a, b, c\}$ and $p=(a b c, a b c, a b c, c a b, c b a)$ be a profile. It is obvious that the Condorcet ranking is $a b c$. It can be verified that $M \operatorname{score}(a, p)=2$, $\operatorname{Mscore}(b, p)=4$, and $\operatorname{Mscore}(c, p)=3$ and hence $\varphi^{\operatorname{Minimax}}(p)=\{a c b\}$. Therefore Minimax does not satisfies Condorcet criterion for welfare rules.

\section{Proofs for Borda rule}

\section{C.1 Borda satisfies $P C W$.}

Proof of Proposition 5. Consider any profile $p \in \mathbb{L}^{N}$. Let $\hat{R} \in \underset{R \in \varphi^{\operatorname{Borda}\left(p_{-i}\right)}}{\operatorname{argmax}} U_{i}(R)$ and $\bar{R} \in \underset{R \in \varphi^{B o r d a}(p)}{\operatorname{argmax}} U_{i}(R)$ for some $i \in N$. 
We first show that $(p(i) \cap \hat{R}) \subseteq(p(i) \cap \bar{R})$. Take any distinct pair of alternatives $a b \in(\hat{R} \cap p(i))$. Since $a b \in \hat{R}$ we have,

$$
\operatorname{Bscore}\left(a, p_{-i}\right) \geq \operatorname{Bscore}\left(b, p_{-i}\right) \text {. }
$$

Also since $a b \in p(i)$ we have,

$$
\operatorname{Bscore}(a, p(i))>\operatorname{Bscore}(b, p(i)) \text {. }
$$

By definition, Bscore $(a, p)=\operatorname{Bscore}\left(a, p_{-i}\right)+B \operatorname{score}(a, p(i))$. Then by Equations 4 and 5 , we have $\operatorname{Bscore}(a, p)>\operatorname{Bscore}(b, p)$ and hence $a b \in \bar{R}$. Since $a b \in p(i)$, we have $(p(i) \cap \hat{R}) \subseteq(p(i) \cap \bar{R})$ which implies,

$$
|p(i) \cap \hat{R}| \leq|p(i) \cap \bar{R}|
$$

Using the definition of the Kemeny distance we have,

$$
\begin{aligned}
& \delta(p(i), \hat{R})=2(m(m-1)-|p(i) \cap \hat{R}|) \\
& \delta(p(i), \bar{R})=2(m(m-1)-|p(i) \cap \bar{R}|)
\end{aligned}
$$

Equation 6, together with the two equations above, yields $\delta(p(i), \bar{R}) \leq \delta(p(i), \hat{R})$. Therefore $U_{i}(\bar{R}) \geq U_{i}(\hat{R})$.

\section{C.2 Borda does not satisfy $C C W$.}

Proof of Proposition 6. Let $A=\{a, b, c\}$ and $p=(a b c, a b c, a b c, c a b, c a b)$ be a profile. It is straight forward to see that $a b c$ is the Condorcet ranking. As $\operatorname{Bscore}(a, p)=8$, Bscore $(b, p)=3$ and Bscore $(c, p)=4$, then $a c b$ is the Borda ranking. Hence Borda does not satisfy $C C W$. 